\title{
ESTOQUES TOTAIS DE CARBONO ORGÂNICO E SEUS COMPARTIME NTOS EM ARGISSOLO SOB FLORESTA E SOB MILHO CULTIVADO COM ADUBAÇÃO MINERAL E ORGÂNICA ${ }^{(1)}$
}

\author{
L. F. C. LEITE (2), E. S. MENDONÇA ${ }^{(3)}, J$. C. L. NE VES ${ }^{(3)}$, \\ P. L. O. A. MACHADO ${ }^{(4)} \&$ J . C. C. GALVÃO(5)
}

\begin{abstract}
RESUMO
Os estoques de matéria orgânica do solo e seus compartimentos são importantes na disponibilidade de nutrientes, agregação do solo e no fluxo de gases de efeito estufa entre a superfície terrestre e a atmosfera. Os objetivos deste estudo foram: (a) avaliar os efeitos de sistemas de produção de milho sob adubação orgânica e mineral nos estoques totais de carbono orgânico (COT) e nitrogênio (NT) e de comparti mentos de carbono (C) orgânico, em um Argissolo Vermelho-Amarelo, e (b) estimar a contri bui ção desses sistemas no seqüestro ou emissão de $\mathrm{CO}_{2}$ atmosférico. Os sistemas de produção, durante 16 anos, constaram de combinações entre dois níveis $(0$ e 1) de composto orgânico, nas doses de 0 e $40 \mathrm{~m}^{3} \mathrm{ha}^{-1}(\mathrm{AO})$, e três níveis $(0,1 \mathrm{e})$ de adubo mineral, nas doses de 0,250 (AM1), e $500 \mathrm{~kg} \mathrm{ha}^{-1}$ (AM2) da fórmula 4-14-8. Uma área sob Floresta Atlântica (FA) adjacente ao experimento foi amostrada e usada como referência de um estado de equilíbrio. Os sistemas de produção em que o composto orgânico foi adicionado apresentaram maiores estoques de COT, NT, carbono da fração leve $\left(\mathrm{C}_{\mathrm{FL}}\right)$ e carbono lábil $\left(\mathrm{C}_{\mathrm{L}}\right)$ do que os sistemas sem adubação ou apenas com adubação mineral, o que confirma a adubação orgânica como estratégia de manejo importante para a melhoria da qualidade do solo. No entanto, no solo sob FA, os estoques de COT, NT e dos compartimentos de C foram maiores do que aqueles observados nos sistemas de produção. Em virtude da maior sensibilidade, os estoques dos compartimentos do $\mathrm{C}_{\mathrm{FL}}$ e do $\mathrm{C}_{\mathrm{L}}$ foram reduzidos em maior intensidade do que os estoques de COT, razão por que podem ser usados como indicadores da interferência antrópica ou das mudanças no manejo sobre o estado da matéria orgânica do solo.
\end{abstract}

Termos de indexação: matéria orgânica do solo, manejo do solo, qualidade de solo, aporte de carbono.

\footnotetext{
(1) Parte da pesquisa realizada para a Tese de Doutorado do primeiro autor, apresentada ao Departamento de Sol os da U niversi dade Federal de Viçosa - UFV. Recebido para publicação em agosto de 2002 e aprovado em maio de 2003.

(2) Pesquisador da Empresa Estadual de Pesquisa Agropecuária da Paraíba - EMEPA-PB. Caixa Postal 275, CEP 58013-290 J oão Pessoa (PB). E-mail: luizfcleite@emepa.org.br

(3) Professor do Departamento de Solos, Universidade Federal de Viçosa - UFV. CEP 36570-000 Viçosa (MG). Bolsista do CNPq. E-mails: esm@mail.ufv.br; jcneves@mail.ufv.br

(4) Pesquisador da Embrapa Solos. Rua J ardim Botânico 1024, CEP 22460-000 Rio deJ aneiro (RJ ). E-mail: pedro@cnps.embrapa.br

(5) Professor do Departamento de Fitotecnia - UFV. E-mail: jgalvão@ufv.br
} 


\author{
SUMMARY: TOTAL STOCKS OF ORGANIC CARBON AND ITS POOLS IN \\ ACRISOLS UNDER FOREST AND UNDER MAIZE CULTIVATED \\ WITH MINERAL AND ORGANIC FERTILIZATION
}

\begin{abstract}
Soil organic matter and its different pools havekey importancein nutrient avai lability, soil aggregation, and in the greenhouse gas fluxes between the earth surface and the atmosphere. The objectives of this study were: a) to evaluate the effect of maize production systems under organic and mineral fertilization on total organic carbon (TOC) and total nitrogen (TN) stocks and on organic carbon pools (C) in an Acrisol; and b) to estimate the contribution of these systems in the atmospheric $\mathrm{CO}_{2}$ sequestration or emission. The production systems induded two levels of organic compost: level 0 (control) and level 1 $\left(40 \mathrm{~m}^{3} \mathrm{ha}^{-1}\right)$; and three level s of mineral fertilizer (0,1 and 2), which correspond to 0,250 (AM1), and $500 \mathrm{~kg} \mathrm{ha}^{-1}$ (AM2) of the 4-14-8 formula. Organic and mineral fertilizer were combined and applied during 16 years. As a reference of thesteady state, soil samples were collected from an adjacent area of the samesoil type, under secondary Atlantic Forest (AF). Whereorganic compost was added, the production systems presented higher organic $\mathrm{C}$ and $\mathrm{N}$ soil storage, light fraction carbon $\left(\mathrm{C}_{\mathrm{LF}}\right)$ and labile carbon $\left(\mathrm{C}_{\mathrm{L}}\right)$ than production systems without fertilization or with mi neral fertilizer only. This confirms thei mportance of organic fertilizer utilization as a management strategy to improve soil quality. However, the soil under AF showed higher values of organic $\mathrm{C}$ and $\mathrm{N}$ storage and carbon pools than soils under production systems. Dueto their higher sensitivity, thestocks of the $\mathrm{C}_{\mathrm{LF}}$ and $\mathrm{C}_{\mathrm{L}}$ were more sever dy decreased than the TOC stocks and thus, may be used as indicators of the anthropogenic impact or theinfluence of management al terations on thesoil organic matter.
\end{abstract}

Index terms: soil organic matter, soil management, soil quality, carbon inputs.

\section{NTRODUÇÃO}

Os estoques de matéria orgânica do solo (MOS) em qualquer agroecossistema são obtidos pela interação dos fatores que deter minam sua formação e aqueles que promovem sua decomposição. A hipótese mais aceita estabelece um declínio no estoque de matéria orgânica após a conversão de florestas nativas em sistemas agrícolas (Houghton et al., 1991). Essa redução pode ser atribuída ao aumento da erosão do solo, aos processos mais acelerados de mineralização da matéria orgânica e oxidação de carbono (C) orgânico do sol o eàs menores quantidades de aportes orgânicos em sistemas manejados comparativamente a florestas nativas.

Em sistemas agrícolas, a dinâmica da MOS pode ser influenciada não só pelo manejo por meio da seleção de culturas e de formas de preparo do solo, mas também pela adição de fertilizantes químicos e materiais orgânicos, que influem positivamentenos processos biológicos de decomposição e mineralização da MOS.

A adição de materiais orgâni cos é fundamental à qualidade do solo, caracterizando-se pela liberação gradativa de nutrientes, que reduz processos como lixiviação, fixação e volatilização, embora dependa essencial mente da taxa de decomposi ção, controlada pela temperatura, umidade, textura e mineralogia do solo, além da composição química do material orgânico utilizado (Zech et al., 1997).

Estudos recentes enfatizam a importância das características químicas para determinar a qualidade do aporte orgânico ea disponibilidade de nutrientes (Palm et al., 2001). E m condições tropicais, são requeridas cerca de 7 e $10 \mathrm{Mg} \mathrm{ha}^{-1} \mathrm{ano}^{-1}$ de resíduos com elevada e baixa relação $C: N$, respectivamente, para manter o teor de $C$ orgânico total no sol o em 1 dag kg-1 (Manfogoya et al., 1997).

Por isso, ouso combinado de fertilizantes químicos e materiais orgânicos tem sido recomendado como manejo alternativo, possibilitando a manutenção de alta produtividade, com estabilidade, principal mente quando o material orgânico aplicado apresenta el evada relação C:N eel evados conteúdos delignina e pol ifenóis, e para regiões onde o uso de fertilizante é recomendado (Fernandes et al., 1997).

Em sua maioria, os estudos sobre o efeito de sistemas demanejo evidenciama pouca sensibilidade da medida do C orgânico total. Como alternativa, tem-se apontado o C da biomassa mi crobiana do solo, representando o compartimento ativo da matéria orgânica do solo e o C da fração leve, referenciando o compartimento lento, como indicadores mais sensíveis aos efeitos do manejo.

Considerando um contínuo de sensibilidade ao manejo, ter-se-íam, numa extremi dade, a medida de 
carbono da biomassa microbiana, bastante variável e sensível, e, na outra, a medida do carbono orgânico total do solo, pouco variável e pouco sensível. A fração leve da matéria orgânica do solo tem-se constituído numa medida de sensibilidade intermediária e, mais importante, que reflete as ações antrópicas (Six et al., 2000).

Em condições tropicais, em que a dinâmica da matéria orgânica é relativamente rápida, são escassos os estudos que visam determinar os efeitos da adubação mineral ou orgânica nos compartimentos da matéria orgânica do solo (Kanchikerimath \& Singh, 2001).

Os objetivos deste estudo foram: quantificar os efeitos de sistemas de produção sob adubação orgânica e mineral nos estoques totais no solo de C eN e dos compartimentos de C orgânico e estimar a contribuição desses sistemas no seqüestro ou emissão de $\mathrm{CO}_{2}$ atmosférico.

\section{MATERIAL E MÉTODOS}

A área do estudo está localizada na Estação Experimental da Universidade Federal de Viçosa, no município de Coimbra (MG) $\left(20^{\circ} 45^{\prime} \mathrm{S}, 42^{\circ}\right.$ 51 'W e 700 m de altitude), na Zona da Mata mineira. A temperatura e a precipitação média anuais são de $19{ }^{\circ} \mathrm{C}$ e $1.400 \mathrm{~mm}$, respectivamente.

Original mente sob vegetação natural até 1930, a área passou a ser cultivada subseqüentemente, por 53 anos, com culturas de subsistência, como o milho e o feijão. O experimento foi iniciado em 1984, com a adequação da área, para estabelecer os sistemas de produção. Durante o período do experimento, foram realizadas duas calagens com calcário dolomítico, para correção do solo. Procedeu-se ao preparo do sol o com aração, utilizando arado de disco reversível e uma gradagem com grade de disco convencional.

O solo da área éum Argissol o Vermelho-Amarelo fase terraço. No início do experimento, o solo apresentava, na camada de $0-20 \mathrm{~cm}, \mathrm{pH}\left(\mathrm{H}_{2} \mathrm{O}\right)$ de 5,9; $\mathrm{Al}, \mathrm{Ca}$ e $\mathrm{Mg}$, iguais a 0; 2,6 e 1,7 $\mathrm{cmol}_{\mathrm{c}} \mathrm{dm}^{-3}$, respectivamente; $\mathrm{P}$ e $\mathrm{K}$ disponíveis (Mehlich- 1 ) iguais a 11 e $58 \mathrm{mg} \mathrm{dm}^{-3}$, respectivamente, e o teor de $C$ orgânico igual a 2,3 dag kg-1 (Walkley Black). Os teores de areia grossa, areia fina, silte e argila foram de $8,6,16$ e $70 \%$, respectivamente, e a densidade do solo igual a 1,1 $\mathrm{Mg} \mathrm{m}^{-3}$ (Galvão, 1988).

O experimento consistiu de adubações, orgânica e mineral, contínuas em cultivo de milho exclusivo, instalado em um arranjo fatorial com parcelas dispostas no del ineamento em blocos casualizados, constituído de seis tratamentos e quatro repetições. Os tratamentos constaram de combinações entre dois níveis ( 0 e 1 ) de composto orgânico, nas doses de 0 (testemunha) e $40 \mathrm{~m}^{3}$ ha-1 (AO), e três níveis $(0,1$ e 2$)$ de adubo mineral, nas doses de 0,250 (AM 1), e $500 \mathrm{~kg} \mathrm{ha}^{-1}$ (AM2) da fórmula 4-14-8. Foram realizadas adubações de cobertura com sulfato de amônio, nas doses de 0, 100 e $200 \mathrm{~kg} \mathrm{ha}^{-1}$, exceto para os tratamentos que continham apenas adubo orgânico. O composto orgânico utilizado, produzido com pal ha de soja e de feijão misturados a esterco bovino, apresentou, em média, $380 \mathrm{~g} \mathrm{~kg}^{-1}$ de água, $0,36 \mathrm{~g} \mathrm{~cm}^{-3}$ de densidade e teores de $\mathrm{P}, \mathrm{K}$, $\mathrm{Ca}, \mathrm{Mg}$ e $\mathrm{N}$ de 0,$7 ; 2,8 ; 1,0 ; 0,4$ e $3,2 \mathrm{dag} \mathrm{kg}^{-1}$, respectivamente. $\mathrm{O}$ valor da $\mathrm{C}: \mathrm{N}$ do composto orgânico foi de cinco. O composto orgânico e o adubo químico foram aplicados no sulco de semeadura e incorporados com enxada.

Uma área sob F loresta Atlântica (FA), adjacente ao experimento (distanteaproximadamente $100 \mathrm{~m}$ ), com a mesma classe de solo, foi usada como referência de estado de equilíbrio. Nesta área, foi definido, no terço médio, um transecto de aproximadamente 100 metros, no qual foram delimitadas quatro subáreas, para obtenção das amostras.

No experimento, foram col etadas, em cada unidade experimental, oito amostras simples com trado manual, para formar uma amostra composta, das camadas de 0-10 e 10-20 cm. Para a área de Floresta Atlântica, foram coletadas 15 amostras simples por área delimitada seguindo as mesmas camadas. Os valores para a camada de $0-20 \mathrm{~cm}$ foram provenientes da média aritmética das camadas amostradas. As amostras de solo foram destorroadas e passadas em peneira de malha de $2 \mathrm{~mm}$, e uma porção em torno de $100 \mathrm{~g}$ (de cada amostra) foi separada, acondicionada e mantida sob refrigeração, entre 4 e $8^{\circ} \mathrm{C}$, visando determinar a biomassa microbiana. Para as demais análises, as amostras de sol o foram secas ao ar (TFSA).

O carbono orgânico total do solo (COT) foi quantificado por oxidação da matéria orgânica via úmida, empregando sol ução de dicromato de potássio em meioácido, com fonte externa de cal or (Yeomans \& Bremner, 1988). O fracionamento químico das substâncias húmicas foi realizado segundo a técnica da solubilidade diferencial, separando-se os ácidos fúlvicos $(F A F)$, os ácidos húmicos $(F A H)$ e as huminas (HUM), de acordo com os conceitos de frações húmicas estabelecidos pela Sociedade Internacional deSubstâncias Húmicas (Swift, 1996). $\mathrm{O}$ extrator utilizado foi $\mathrm{NaOH} 0,1 \mathrm{~mol} \mathrm{~L}^{-1}$. A determinação do carbono dessas frações foi efetuada segundo Yeomans \& Bremner (1988).

O carbono orgânico lábil $\left(C_{L}\right)$ foi quantificado por meio de oxidação com $\mathrm{KMnO}_{4}\left(33 \mathrm{mmol} \mathrm{L}^{-1}\right)$, como proposto por Blair et al. (1995) e modificado por Shang \& Tiessen (1997), enquanto o carbono nãolábil $\left(C_{N L}\right)$, equivalente ao $C$ não oxidado pelo $\mathrm{KMnO}_{4}$, foi determinado por diferença $\left(\mathrm{C}_{\mathrm{NL}}=\mathrm{COT}-\right.$ $\mathrm{C}_{\mathrm{L}}$ ). 
Com basenas mudanças no COT, entre um sistema referência e um sistema cultivado, foi criado um Índice de Compartimento de Carbono (ICC), calculado como: ICC = COT $_{\text {cultivado }} / \mathrm{COT}_{\text {referência. }}$. Com basenas mudanças na proporção de $C_{L}$ (i.e. $L=C_{L} / C_{N L}$ ) no solo, um Índice de Labilidade (I L) foi determinado como : IL $=\mathrm{L}_{\text {cultivado }} \mathrm{L}_{\text {referência. }}$ Estes dois índices foram usados para calcular o Índice de Manejo de Carbono (I MC), obti do pela seguinte expressão: I MC $=$ ICC x IL x 100 (Blair et al., 1995).

O nitrogênio total (NT) foi quantificado nas amostras de solo submetidas à digestão sulfúrica e dosado por destilação Kjeldhal (Bremner, 1996). O carbono da biomassa microbiana $\left(C_{M I C}\right)$ foi determinado pelo método da irradiação-extração, utilizando forno de microondas (Cônsul, freqüência de $2.450 \mathrm{MHz}$, energia a $900 \mathrm{~W}$ por 180 segundos) (I slam \& Weil, 1998). O extrator utilizado foi $\mathrm{K}_{2} \mathrm{SO}_{4}$ $0,5 \mathrm{~mol} \mathrm{~L}^{-1}$ e o carbono contido nos extratos foi quantificado por meio de procedimento por oxidação úmida (Yeomans \& Bremner, 1988). O fator de conversão $\left(K_{C}\right)$ usado para converter o fluxo de $C$ para $\mathrm{C}$ da biomassa microbiana foi de 0,33 (Sparling $\&$ West, 1988). A proporção $C_{\text {MIC }} / C O T$ ou quociente microbiano foi calculado para refletir os aportes de C e a conversão de substratos orgânicos para o C da biomassa microbiana (Sparling, 1992).

A fraçãoleve-livrefoi determinada por flotaçãoem solução de iodeto de sódio, densidade de $1,8 \mathrm{~g} \mathrm{~cm}^{-3}$, como proposto por Sohi et al. (2001). O material obtido foi seco sob temperatura de $80^{\circ} \mathrm{C}$, por $72 \mathrm{~h}$. $\mathrm{O}$ carbono da fração leve $\left(\mathrm{C}_{\mathrm{FL}}\right)$ foi quantificado por combustão via seca em analisador Perkin Elmer CHNS/O 2400.

Os valores de densidade do solo, apresentados por Galvão (1988) e determinados neste trabalho, foram utilizados para cal cular os estoques de COT e NT e de compartimentos de C numa equivalência com a massa de solo (Angers et al., 1997; Carter et al., 1998; Leite, 2002). Os estoques do COT no início do experimento, em 1984 (46 $\mathrm{Mg} \mathrm{ha}^{-1}$ ) eno ano 2000 , foram usados para calcular a contribuição dos sistemas de produção no seqüestro ou na emissão de $\mathrm{C}-\mathrm{CO}_{2}$ pelo solo, na camada de $0-20 \mathrm{~cm}$, considerando o fator de conversão de $\mathrm{C}$ para $\mathrm{CO}_{2}$ de 3,67 (massa molar do $\mathrm{CO}_{2}$ /massa molar do $\mathrm{C}$ ). O tempo de residência médio (TRM) do COT foi calculado como o inverso da constante de decomposição $(1 / k)$, assumindo que o $C$ declina a partir do $C$ da Floresta Atlântica $\left(C_{F A}\right)$ seguindo a equação $C=C_{F A} e^{-k t}$, em que t é o tempo em anos.

Os dados de estoques de COT e NT e dos compartimentos de carbono nos sistemas de produção foram submetidos à análise de variância, num delineamento de blocos casualizados, com quatro repetições. A comparação das médias foi feita com base na significância do teste $F$, considerando as camadas de 0-10, 10-20 e 0-20 cm isoladamente, com auxílio do sistema computacional SAEG (UFV,
1993). Para a área de FA, procedeu-se à análise estatística quantitativa descritiva de média e desviopadrão.

\section{RESULTADOS E DISCUSSÃO}

\section{Estoques totais de carbono e nitrogênio no solo após 16 anos}

Os sistemas de produção com adubação orgânica resultaram em maiores estoques $(P<0,05)$ de carbono orgânico total (COT) do que os sistemas sem adubação ou apenas com adubação mineral (Quadro 1). Nas camadas de $0-10 \mathrm{~cm}$ e de $10-20 \mathrm{~cm}$, os estoques variaram de 18,87 (0) a $26,50 \mathrm{Mg} \mathrm{ha}^{-1}$ (AM 1 + AO) e de 18,04 (0) a 24,81 $\mathrm{Mg} \mathrm{ha}^{-1}$ $(A M 1+A O)$, respectivamente.

Os estoques denitrogênio total (NT) apresentaram tendência similar ao COT, variando de 1,54 (0) a $2,11 \mathrm{Mg} \mathrm{ha}^{-1}(\mathrm{AM} 1+\mathrm{AO})$, na camada superficial, e de 1,53 (0) a 2,02 $\mathrm{Mg} \mathrm{ha}^{-1}$ (AM 1 + AO), na camada mais profunda. O aumento dos estoques de COT e NT nos sistemas que receberam adubação orgânica está provavelmente associado ao maior aporte de resíduos vegetais retornado ao solo $\left(0: 5,4 \mathrm{Mg} \mathrm{ha}^{-1}\right.$; AM 1: 9,0 Mg ha-1; AM 2: 11,8 $\mathrm{Mg} \mathrm{ha}^{-1} ; A O$ : 13,4 $\mathrm{Mg} \mathrm{ha}^{-1}$; AM 1+AO: $15,4 \mathrm{Mg} \mathrm{ha}^{-1} \mathrm{AM} 2$ + AO: 16,7 Mg ha-1; Galvão, 1995; Leite, 2002), como observado em diversos estudos (Omay et al., 1997; Kanchikerimath \& Singh, 2001), e à aplicação de $1,4 \mathrm{Mg} \mathrm{ha}^{-1}$ ano-1 $^{-1}$ de C por meio do composto orgânico. Embora a adubação orgânica tenha aumentado significativamente os estoques de COT, parte deste aumento reflete apenas o $\mathrm{C}$ importado de outro compartimento.

Após 16 anos de cultivo, todas as estratégias de manejo resultaram em redução nos estoques deCOT em relação à Floresta Atlântica (FA), indicando a susceptibilidade da oxidação do COT dos solos sob vegetação natural, quando estes são submetidos à agricultura. Na camada de $0-20 \mathrm{~cm}$, os estoques de COT foram reduzidos em 42, 38, 37, 25, 20 e $22 \%$, para 0, AM1, AM2, AO, AM 1 + AO e AM2 + AO, respectivamente (Quadro 1 ). Com exceção da testemunha, os outros sistemas de produção apresentaram redução nos estoques de COT comparado à FA menor do que o percentual de $40 \%$ estimado por Scholes et al. (1997) para solos tropicais.

As menores perdas de COT foram observadas nos sistemas submeti dos à adubação orgânica, de forma similar aos resultados obtidos nos experimentos clássicos de Rothamsted (J enkinson, 1991) e em experimentos delonga duração (26 anos) no Quênia (Kapkiyai et al., 1999) e na Î́ndia (Kanchikerimath \& Singh, 2001), indicando maior resiliência destes sistemas. O tempo de residência médio (TRM) do C, maior nos sistemas de produção com adubação 
Quadro 1. Estoques totais de carbono orgânico (COT) e nitrogênio (NT) nas profundidades de 0-10 cm, $10-20 \mathrm{~cm}$ e 0-20 cm, de um Argissolo Vermelho-Amarelo, de acordo com as adubações, mineral e orgânica, e em Floresta Atlântica

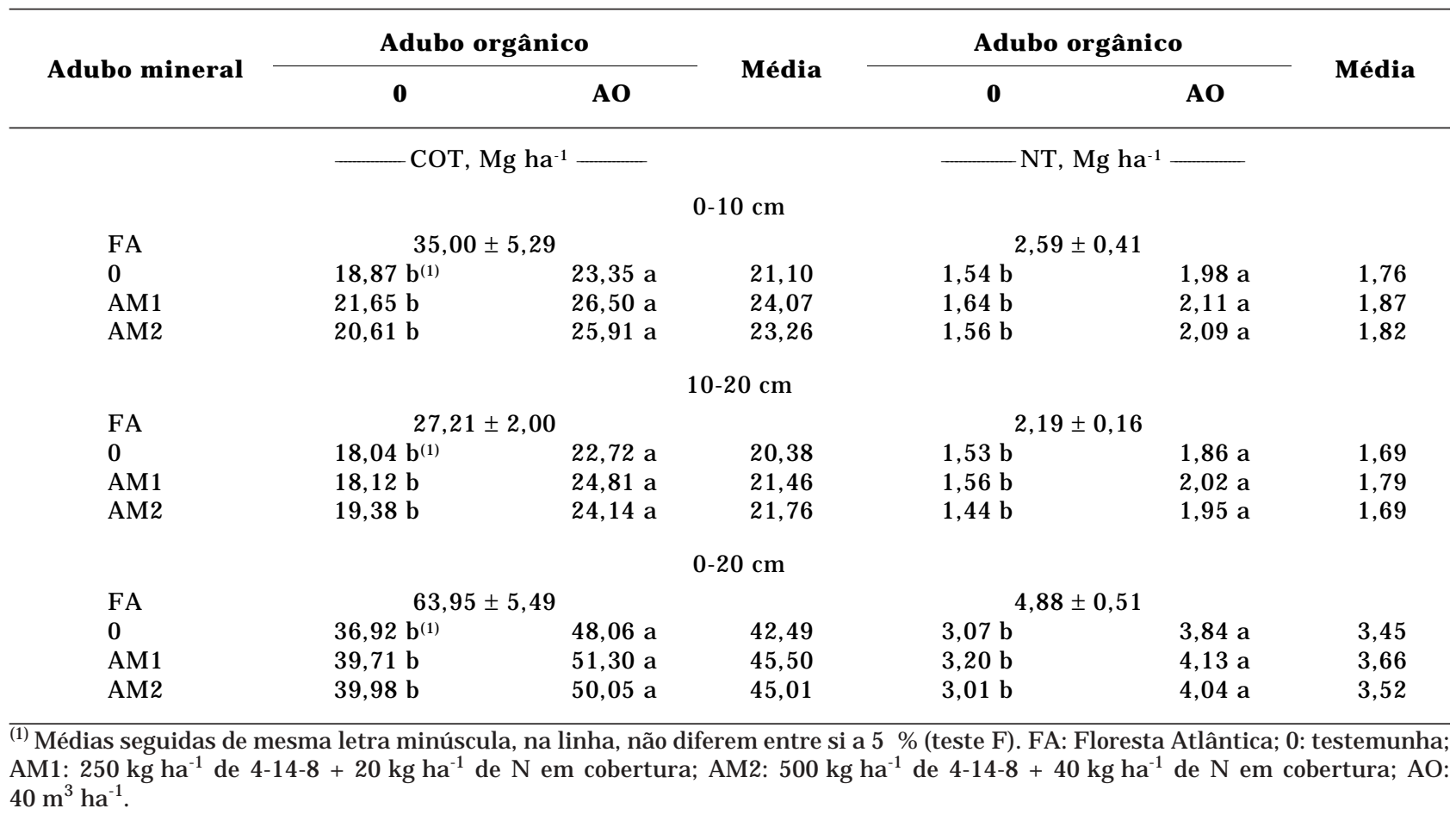

orgânica do que nos sistemas apenas com adubação mineral ou sem adubação, confirma esta tendência (Quadro 2). Além disso, se forem considerados os estoques de COT no início do experimento $\left(46 \mathrm{Mg} \mathrm{ha}^{-1}\right)$, os sistemas de produção com a presença da adubação orgânica resultaram em aumento desses estoques ao longo de 16 anos.

Quadro 2. Tempo de residência médio do C (TRM), estimativa de emissão de $\mathrm{C}-\mathrm{CO}_{2}$ e taxa anual de emissão de $\mathrm{C}_{-} \mathrm{CO}_{2}$, na camada de $0-20 \mathrm{~cm}$ de um Argissolo Vermelho-Amarelo, de acordo com as adubações, mineral e orgânica, após 16 anos

\begin{tabular}{lccc}
\hline Sistema & TRM & Emissão C-C $\mathbf{C}_{\mathbf{2}}$ & Taxa C-C O \\
\hline & ano & M g ha-1 & ${\text { M g ha-1 } \text { ano }^{-1}}^{-1}$ \\
0 & 29 & 81,40 & 5,09 \\
AM 1 & 34 & 102,42 & 6,40 \\
AM 2 & 34 & 125,78 & 7,86 \\
AO & 56 & 136,14 & 8,51 \\
AM 1 + AO & 73 & 141,89 & 8,87 \\
AM 2 + AO & 65 & 157,18 & 9,82
\end{tabular}

0: testemunha; AM 1: $250 \mathrm{~kg} \mathrm{ha}^{-1}$ de 4-14-8 +20 kg ha-1 de N em cobertura; AM2: $500 \mathrm{~kg} \mathrm{ha}^{-1}$ de 4-14-8 + $40 \mathrm{~kg} \mathrm{ha}^{-1}$ de N em cobertura; AO: adubo orgânico, $40 \mathrm{~m}^{3} \mathrm{ha}^{-1}$.
A perda de COT em sistemas agrícolas deveu-se à intensificação da atividade biológica provocada pel o revolvimento do solo, pela correção da acidez e pela adubação, o que cria um ambiente mais favorável à ação dos microrganismos, em razão do incremento do teor de água em profundidade, das melhores condições de arejamento e da disponibilidade denutrientes (Paustian et al., 1997). Os estoques de NT também foram maiores no solo sob FA comparado ao solo sob os sistemas de produção. Na camada de $0-20 \mathrm{~cm}$, as perdas de NT variaram de 37 (0) a 15,4\% (AM1 + AO), significando, de forma semel hanteaoCOT, menores perdas com a presença da adubação orgânica.

\section{Estimativa de seqüestro ou emissão de $\mathrm{C}-\mathrm{CO}_{2}$ atmosférico pelos sistemas de produção}

Os estoques de $C$ são determinados pel o balanço das entradas, como o aporte de resíduos vegetais ea aplicação de compostos orgânicos, e as saídas, por meio da decomposição da matéria orgânica do solo. Por isso, para se ter seqüestro de $\mathrm{C}-\mathrm{CO}_{2}$, é essencial o aumento das entradas de C, a diminuição da decomposição, ou ambos. N este estudo, o aporte de C foi mai or nos sistemas de produção com adubação orgânica, o que poderia indicar maior potencial para seqüestro de $\mathrm{C}-\mathrm{CO}_{2}$.

No entanto, após 16 anos de cultivo, os sistemas de produção com adubação orgânica emitiram em 
torno de 1,5 vez mais $\mathrm{C}-\mathrm{CO}_{2}$ do que aqueles sem a presença da adubação orgânica (Quadro 2). As maiores taxas de emissão de $\mathrm{C}-\mathrm{CO}_{2}$ foram calculadas no sistema AM2 + AO $\left(9,82 \mathrm{Mg} \mathrm{ha}^{-1} \mathrm{ano}^{-1}\right.$ deC $\left.-\mathrm{CO}_{2}\right)$ eas menores na testemunha $\left(5,09 \mathrm{Mg} \mathrm{ha}^{-1} \mathrm{ano}^{-1} \mathrm{deC}-\mathrm{CO}_{2}\right)$.

O composto orgânico usado neste estudo, parcialmente constituído por esterco animal, apresenta elevada proporção de materiais recalcitrantes, pois os compostos lábeis são seletivamente removidos no processo de digestão (Paustian e al., 1997). Por isso, nem todo ganho de COT, a partir das aplicações do composto, representa remoção líquida de $\mathrm{CO}_{2}$ atmosférico; a maioria é apenas uma redistribuição do $C$ proveniente do material vegetal usado como forragem, indicando que a adubação orgânica não seqüestra $\mathrm{C}-\mathrm{CO}_{2} \mathrm{em}$ solos, como pressuposto em alguns estudos (Swift, 2001) e preconizado no Protocolo de Quioto (artigo 3.3). Entretanto, por meio dos benefícios causados à fertilidade e à estrutura do solo, as aplicações dos compostos orgânicos podem aumentar o rendimento das culturas e, portanto, contribuir para ganhos de $\mathrm{C}$ a partir de maior aporte de resíduos vegetais.

\section{Estoques dos compartimentos de carbono orgânico}

Os estoques de carbono da biomassa microbiana $\left(\mathrm{C}_{\text {MIC }}\right)$ foram similares nos sistemas de produção com adubação orgânica e adubação mineral, ou sem adubação (Quadro 3). I sto pode ser devido à recal citrância do $C$ no composto orgânico aplicado. A amostragem do $C_{\text {MIC }}$ foi realizada após a colheita da cultura do milho e previamente à incorporação dos resíduos vegetais, o que pode indicar a ausência nos sistemas de manejo de el evada proporção de C facilmente disponível, que favorece o aumento da biomassa microbiana, conforme constatado por Six et al. (1998) e Kanchikerimath \& Singh (2001) em experimentos com adubação orgânica e mineral .

Como os aportes de resíduos nos sistemas que receberam adubação or gânica foram mai ores do que os dos sistemas apenas com adubação mineral, ou sem adubação, poder-se-ia estimar que o $\mathrm{C}_{\text {MIC }}$, avaliado em diversos períodos, provavel menteseria maior no sistema de produção com adubação orgânica.

O carbono da fraçãoleve $\left(C_{F L}\right)$ foi maior $(P<0,05)$ nos sistemas de produção com adubação orgânica do que nos sistemas apenas com adubação mineral ou sem adubação (Quadro 3). N as camadas de 0-10 e de $10-20 \mathrm{~cm}$, os mai ores estoques foram observados nos sistemas AM $1+A O\left(2,02 \mathrm{Mg} \mathrm{ha}^{-1}\right)$ e $\mathrm{AO}$ e $A M 1+A O\left(1,69 \mathrm{Mg} \mathrm{ha}^{-1}\right)$, respectivamente. Os maiores estoques de $C_{F L}$ nos sistemas com adubação orgânica provavel mente são decorrentes das mai ores produtividades das culturas nestes sistemas (dados não mostrados), o que causa aumento no retorno para o sol o desubstratos orgânicos, por meio da parte aérea, raízes e exsudatos, em relação aos sistemas sem a presença de adubação orgânica.

Quadro 3. Estoques de carbono da biomassa microbiana $\left(C_{\mathrm{MIC}}\right)$ e de carbono da fração leve $\left(\mathrm{C}_{\mathrm{FL}}\right)$, nas profundidades de 0-10, 10-20 e 0-20 cm, de um Argissolo Vermelho-Amarelo, de acordo com as adubações, mineral e orgânica, e em Floresta Atlântica

\begin{tabular}{|c|c|c|c|c|c|c|}
\hline \multirow{2}{*}{ Adubo mineral } & \multicolumn{2}{|c|}{ Adubo orgânico } & \multirow{2}{*}{ Média } & \multicolumn{2}{|c|}{ Adubo orgânico } & \multirow{2}{*}{ Média } \\
\hline & $\mathbf{0}$ & AO & & $\mathbf{0}$ & AO & \\
\hline & \multicolumn{2}{|c|}{$\mathrm{C}_{\mathrm{MIC}}, \mathrm{Mg} \mathrm{ha}^{-1}$} & & \multicolumn{2}{|c|}{$-\mathrm{C}_{\mathrm{FL}}, \mathrm{Mg} \mathrm{ha} \mathrm{A}^{-1}$} & \\
\hline \multicolumn{7}{|c|}{$0-10 \mathrm{~cm}$} \\
\hline FA & \multicolumn{2}{|c|}{$0,90 \pm 0,19$} & & \multicolumn{2}{|c|}{$11,74 \pm 0,31$} & \\
\hline 0 & $0,30 a$ & $0,34 a$ & 0,32 & $1,39 b^{(1)}$ & 1,99 a & 0,69 \\
\hline AM 1 & $0,35 a$ & $0,47 a$ & 0,41 & $1,33 \mathrm{~b}$ & 2,02 a & 0,67 \\
\hline AM 2 & $0,36 \mathrm{a}$ & $0,42 a$ & 0,39 & $1,21 \mathrm{~b}$ & $1,89 \mathrm{a}$ & 1,55 \\
\hline \multicolumn{7}{|c|}{$10-20 \mathrm{~cm}$} \\
\hline FA & \multicolumn{2}{|c|}{$0,65 \pm 0,10$} & \multicolumn{4}{|c|}{$5,02 \pm 057$} \\
\hline 0 & $0,26 a$ & 0,31 a & 0,28 & $1,09 b^{(1)}$ & 1,69 a & 0,39 \\
\hline AM 1 & $0,28 a$ & 0,32 a & 0,30 & $1,24 a$ & 1,69 a & 0,46 \\
\hline AM2 & $0,31 \mathrm{a}$ & $0,39 a$ & 0,35 & $1,08 \mathrm{~b}$ & $1,68 \mathrm{a}$ & 0,38 \\
\hline \multicolumn{7}{|c|}{$0-20 \mathrm{~cm}$} \\
\hline FA & \multicolumn{2}{|c|}{$1,59 \pm 0,26$} & \multicolumn{4}{|c|}{$17,76 \pm 0,86$} \\
\hline 0 & $0,57 a$ & $0,65 \mathrm{a}$ & 0,61 & $2,48 b^{(1)}$ & 3,68 a & 3,08 \\
\hline AM 1 & $0,63 a$ & $0,79 a$ & 0,71 & $2,57 \mathrm{~b}$ & 3,71 a & 3,14 \\
\hline AM 2 & $0,68 \mathrm{a}$ & $0,80 \mathrm{a}$ & 0,74 & $2,29 \mathrm{~b}$ & $3,57 \mathrm{a}$ & 2,93 \\
\hline
\end{tabular}

(1) Médias seguidas de mesma letra minúscula, na linha, não diferem entre si a 5 \% (teste F). FA: Floresta Atlântica; 0: testemunha; AM 1: $250 \mathrm{~kg} \mathrm{ha}^{-1}$ de 4-14-8 + $20 \mathrm{~kg} \mathrm{ha}^{-1}$ de N em cobertura; AM2: $500 \mathrm{~kg} \mathrm{ha}^{-1}$ de 4-14-8 +40 kg ha-1 de $\mathrm{N}$ em cobertura; $\mathrm{AO}: 40 \mathrm{~m}^{3} \mathrm{ha}^{-1}$. 
Os estoques de $C_{\text {MIC }}$ nos sistemas de produção 0 , $A M 1, A M 2, A O, A M 1+A O, A M 2+A O$ foram menores do que os da FA, em 67, 61, 60, 62, 48 e $47 \%$, na camada de $0-10 \mathrm{~cm}$, e em $60,57,52,52,51$ e $40 \%$, na camada de $10-20 \mathrm{~cm}$ (Quadro 3), o que pode estar relacionado, especialmente nos sistemas com adubação orgânica, ao longo período de manejo convencional, desde a derrubada da FA até o início do experimento.

Essas perdas em relação à $F A$ foram maiores do que aquelas observadas para oCOT, mostrando que o $C_{\text {MIC }}$ responde mais rapidamenteàs alterações nas práticas de manejo. Em modelos de simulação da dinâmica da matéria orgânica do solo, como o Century (Parton et al., 1987), o $\mathrm{C}_{\text {MIC }}$ é relacionado com o compartimento ativo e apresenta curto tempo de ciclagem que varia de 1 a 2 anos. Por outrolado, as variações do $C_{\text {MIC }}$ podem estar também associadas às condições diferenciadas de temperatura e umidade existentes na FA e nos sistemas de produção.

A proporção $C_{\text {MIC }} / C O T$, ou quociente microbiano, um indicador da disponibilidade da matéria orgânica para os microrganismos, foi maior no solo sob FA, tanto na camada de $0-10 \mathrm{~cm}(2,57 \%)$, quanto na camada de $10-20 \mathrm{~cm}(2,36 \%)$. Nos sistemas de produção, a variação foi de 1,3 (AO) a 1,77 \% (AM $1+A O)$, na camada de $0-10 \mathrm{~cm}$, e de 1,3 $(\mathrm{AM} 1+\mathrm{AO})$ a 1,60 \% (AM2 + AO), na camada de 10$20 \mathrm{~cm}$ (Quadro 4). Esses valores estão na mesma ordem de magnitude daqueles observados em diversos sistemas agrícolas (1-2\%) e variam de acordo com o clima, pH, sistemas de cultura e de preparo de solo, quantidade e qualidade do aporte de C (Lutzow et al., 2002).

Os menores valores da proporção $\mathrm{C}_{\mathrm{MIC}} / \mathrm{COT}$ nos sistemas de produção com adubação orgânica contrariam os resultados observados em outros trabal hos (Witter \& Kanal, 1998) e indicam menor disponibilidade de substrato para os mi crorganismos nestes sistemas. Desconhece-se, porém, seesta baixa disponibilidade foi resultado da qualidade do substrato, ou, especificamente, do composto orgânico aplicado, ou, ainda, da formação de complexos organominerais, por meio do efeito protetor da argila. No entanto, como o teor de argila é similar em todos os sistemas, a primeira explicação parece ser mais adequada.

As maiores perdas dos estoques de $C_{F L}$ em relação à $\mathrm{FA}$ foram observadas nos sistemas de produção sem a presença de adubação orgânica. Na camada de $0-10 \mathrm{~cm}$ e de $10-20 \mathrm{~cm}$, as reduções variaram de 83 (AO e AM1 + AO) a $90 \%(A M 2)$ e de $66 \%$ (AO, $\mathrm{AM} 1$ + AO eAM 2 + AO) a $78 \%(0)$ (Quadro 3). Esses resultados indicam maior sensibilidade dos níveis de $C_{F L}$ aos efeitos das alterações nas práticas de manejo do que o COT e o $\mathrm{C}_{\text {MIC }}$ e ressaltam a sua importância na avaliação da degradação da matéria orgânica do solo. A rápida mineralização do $C_{F L}$ é
Quadro 4. Proporção de carbono da biomassa microbiana no carbono orgânico total ( $C_{\text {MIC }}$ COT), do carbono da fração leve no carbono orgânico total $\left(\mathrm{C}_{\mathrm{FL}} / \mathrm{COT}\right)$ e do carbono da bi omassa mi crobiana no carbono da fração leve $\left(C_{\mathrm{MIC}} / \mathrm{C}_{\mathrm{FL}}\right)$, nas profundidades de 0-10, 10-20 e 0-20 cm, de um Argissolo Vermelho-Amarelo, de acordo com as adubações, mineral e orgânica

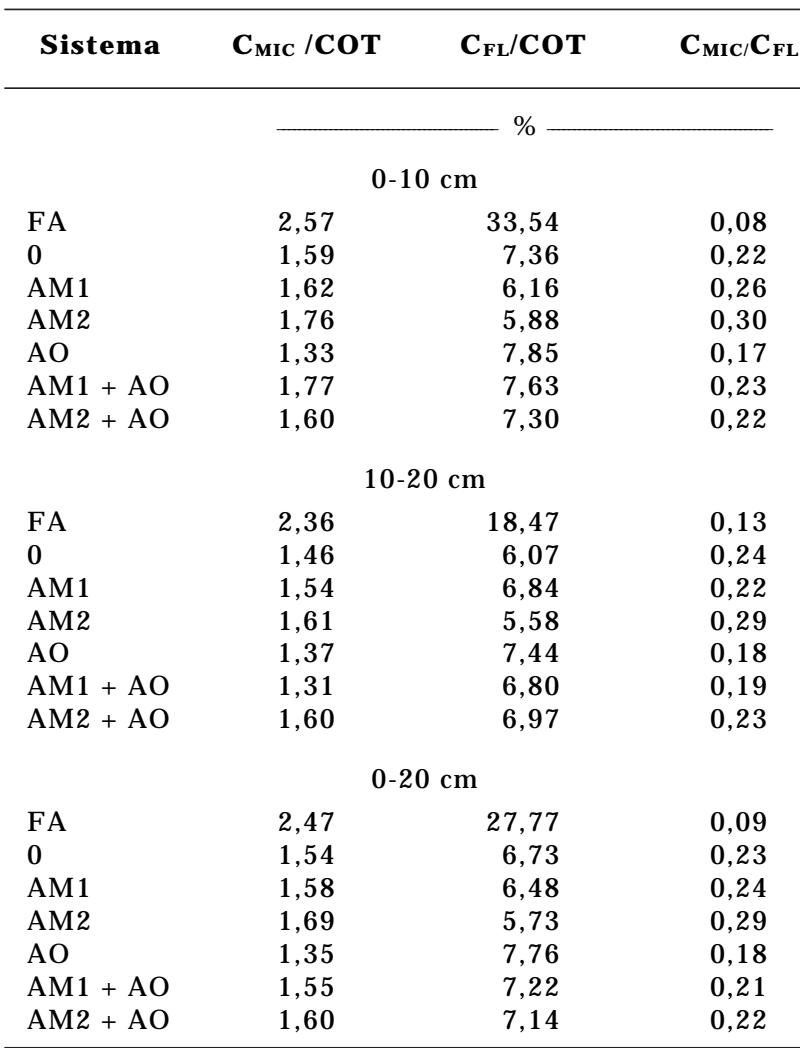

0: testemunha; AM 1: $250 \mathrm{~kg} \mathrm{ha}^{-1}$ de 4-14-8 +20 kg ha-1 de N em cobertura; AM2: $500 \mathrm{~kg} \mathrm{ha}^{-1}$ de 4-14-8 + $40 \mathrm{~kg} \mathrm{ha}^{-1}$ de N em cobertura; AO: adubo orgânico, $40 \mathrm{~m}^{3} \mathrm{ha}^{-1}$; FA: Floresta Atlântica.

provavelmente devida à natureza lábil dos seus constituintes e à falta de proteção física por meio dos agregados. Os estoques maiores de $\mathrm{C}_{\mathrm{FL}}$ verificados na FA podem estar relacionados com o maior aporte de resíduos verificados em sistemas naturais, com a qualidade destes resíduos e com a maior reciclagem dosistema radicular, especial mente das raízes finas (Six et al., 1998).

O $C_{F L}$ representou de 5,88 (AM2) a 7,85 \% (AO) do COT, na camada de $0-10 \mathrm{~cm}$. Na camada de 10$20 \mathrm{~cm}$, a tendência foi similar à camada superficial e a proporção do $C_{F L}$ no COT foi de 5,58 (AM2) a $7,44 \%$ (AO) (Quadro 4). De forma contrária ao quociente mi crobiano, os mai ores percentuais do $\mathrm{C}_{\mathrm{FL}}$ no COT foram observados nos sistemas com adubação orgânica, o que está associado ao maior aporte de resíduos vegetais verificados nestes sistemas. A proporção de $\mathrm{C}_{\mathrm{FL}}$ no COT da Floresta 
Atlântica foi cerca de 5 e 3 vezes aquela obtida pel os sistemas de produção, nas camadas de 0-10 e 10$20 \mathrm{~cm}$, respectivamente. No entanto, o $\mathrm{C}_{\mathrm{FL}}$ é um compartimento transitório que se situa entre os resíduos vegetais e a matéria orgânica humificada eseu tamanho em relação ao COT depende da época de aplicação dos resíduos vegetais e de sua taxa de decomposição (Gregorich \& J anzen, 1996).

No model o Century, o $C_{F L}$, ou a matéria orgânica particulada, constitui o compartimento lento, com ciclagem intermediária entre o compartimento ativo $\left(C_{\text {MIC }}\right)$ e o compartimento passivo. Os valores da proporção $C_{M I C} / C_{F L}$ foram maiores nos sistemas sem a presença da adubação orgânica em todas as camadas, o que indica maior requerimento de substrato por parte dos microrganismos desses sistemas (Quadro 4).

Os maiores estoques $(P<0,05)$ da fração ácidos húmicos (FAH) foram observados nos sistemas de produção com adubação orgânica e variaram de 2,01 (0) a 3,53 Mg ha-1 (AM2 + AO) e de 1,86 (0) a 3,61 Mg ha-1 (AM2 + AO), nas camadas de 0-10 e 10$20 \mathrm{~cm}$, respectivamente (Quadro 5). Os estoques da fração huminas (HUM) foram maiores $(P<0,05)$ apenas no sistema AM2 + AO, quando comparados aos do sistema AM2, nas camadas de $10-20$ e 0-20 cm (Quadro 5).

Os estoques da fração ácidos fúlvicos $(F A F)$ nos sistemas de produção foram menores do que os da
FA (Quadro 5). Nas camadas de 0-10 e 10-20 cm, as reduções variaram de 3,4\% (AMI + AO) a $19 \%$ (AM2) e de 6,5\% (AM2 + AO) e a $17 \%$ (AM1), respectivamente. No entanto, na camada mais profunda, o sistema AM $1+A O$ apresentou estoque da FAF (3,69 $\left.\mathrm{Mg} \mathrm{ha}^{-1}\right)$ similar ao da FA (3,65 $\left.\mathrm{Mg} \mathrm{ha}^{-1}\right)$. Os sistemas de produção que receberam adubação orgânica apresentaram os maiores estoques e, portanto, as menores perdas.

As diminuições nos estoques da FAH nos sistemas de produção foram de maior magnitude do que aquelas demonstradas na FAF. Na camada de $0-$ $10 \mathrm{~cm}$, a menor redução observada foi no sistema AM2 + AO (4,5\%) e a maior na testemunha (46\%). $\mathrm{Na}$ camada de $10-20 \mathrm{~cm}$, os sistemas AM 1 + AO $\left(3,4 \mathrm{Mg} \mathrm{ha}^{-1}\right)$ eAM $2+\mathrm{AO}\left(3,6 \mathrm{Mg} \mathrm{ha}^{-1}\right)$ apresentaram estoques superiores aos da FA $\left(3,15 \mathrm{Mg} \mathrm{ha}^{-1}\right)$. Por outro lado, na testemunha, foi observada a maior redução nos estoques da FAH (41\%). Os estoques da FAH nos sistemas sem a adubação orgânica diminuíram com aumento da profundidade, deforma oposta aos estoques nos sistemas com a presença da adubação orgânica.

Deforma similar às FAF eFAH, a fração huminas (HUM) apresentou menores estoques em relação à FA. Na camada de 0-10 cm, as maiores reduções foram de $34 \%$ e observadas nos sistemas sem a presença de adubação orgânica (0 e AM2). Essa tendência se repetiu na camada de $10-20 \mathrm{~cm}$, com a

Quadro 5. Estoques de carbono nas frações ácidos fúlvicos (FAF), ácidos húmicos (FAH) e humi nas (HUM), nas profundidades de 0-10, 10-20 e 0-20 cm, de um Argissolo Vermelho-Amarelo, de acordo com as adubações, mineral e orgânica, e em Floresta Atlântica

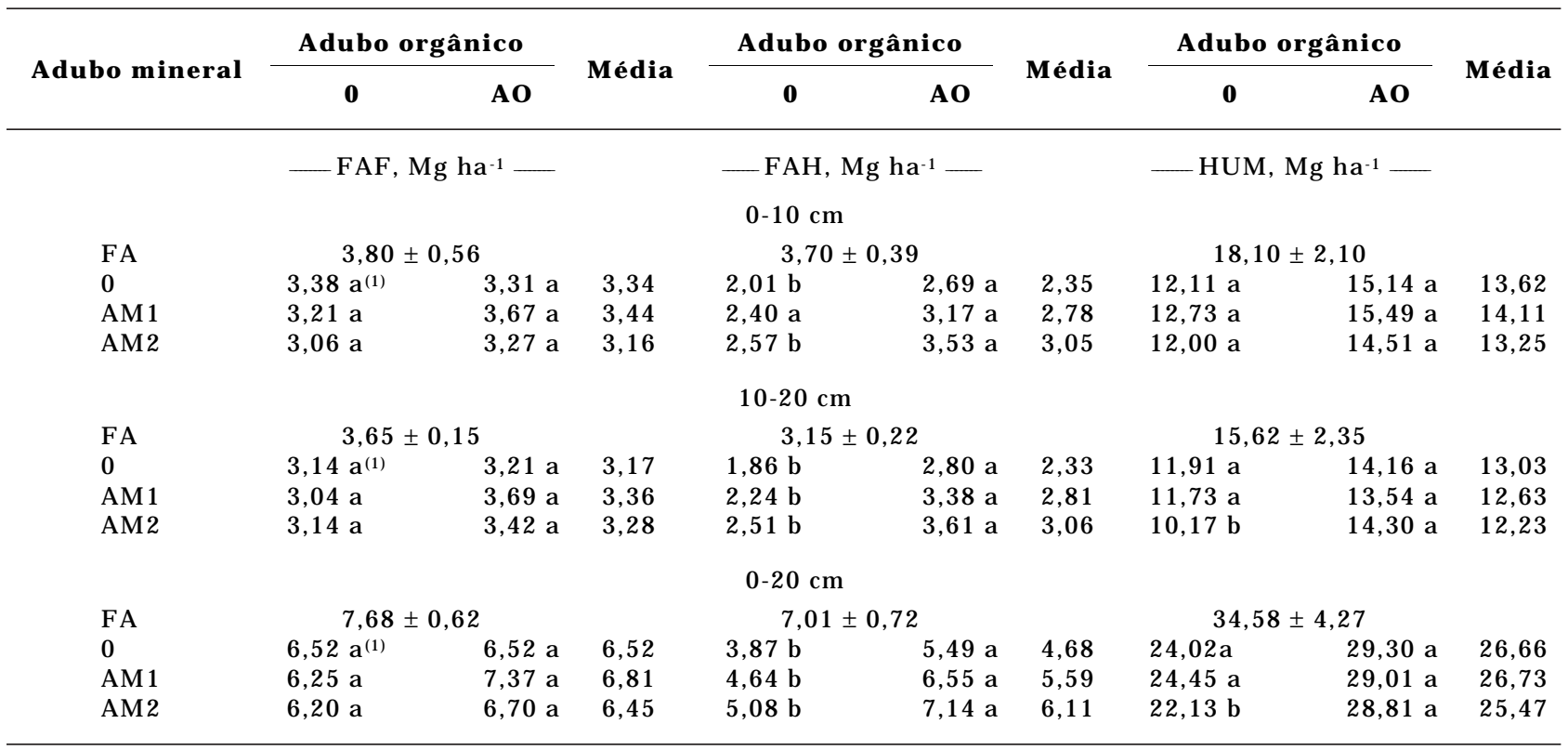

(1) Médias seguidas de mesma letra minúscula, na linha, não diferem entre si a 5 \% (teste F). FA: Floresta Atlântica; 0: testemunha; AM 1: $250 \mathrm{~kg} \mathrm{ha}^{-1}$ de 4-14-8 + $20 \mathrm{~kg} \mathrm{ha}^{-1}$ de $\mathrm{N}$ em cobertura; $\mathrm{AM} 2: 500 \mathrm{~kg} \mathrm{ha}^{-1}$ de 4-14-8 + $40 \mathrm{~kg} \mathrm{ha}^{-1}$ de $\mathrm{N}$ em cobertura; $\mathrm{AO}$ : $40 \mathrm{~m}^{3} \mathrm{ha}^{-1}$. 
maior redução sendo observada no sistema AM2 (35\%). A diminuição dos estoques das frações FAF , FAH e HUM, decorrente da mudança de vegetação natural para agricultura, também foi observada por Ferreira (1997) e Longo \& Espíndola (2000).

A fração HUM constituiu-se de cerca de 50 a $60 \%$ do COT em todos sistemas de produção, similarmente ao observado por Rosales et al. (1999) neste mesmo experimento, o que pode estar relacionado com o tamanho das moléculas e ao longo tempo de residência no solo, associados a esta fração. Por outro lado, as FAF e FAH, por apresentarem menor estabilidade, sofreram processos de movimentação no perfil, polimerização, ou mineralização, diminuindo sua composição percentual no solo. A proporção $F A H / F A F$ foi maior no solo sob $F A$ do que no sol o sob sistemas de produção (Quadro 5).

Em florestas naturais, sem perturbação do solo, percebeu-se maior polimerização de compostos húmicos, aumentando a proporção da $\mathrm{FAH}$ em relação à FAF. Além disso, o maior acúmulo de matéria orgânica do solo nestes sistemas naturais pode contribuir para aumento dos estoques de ácidos húmicos, por meio de processo de herança de compostos da matéria orgânica fresca, de modo semel hante ao que ocorreria com a humina herdada, particularmente, da lignina (Stevenson, 1994).

Dentre os sistemas de produção, a aplicação do composto orgânico em cada cultivo favoreceu a maior proporção dos componentes de maior peso mol ecular, o que pode indicar a presença de matéria orgânica de maior estabilidade.

Os estoques de carbono lábil $\left(C_{L}\right)$ foram maiores $(P<0,05)$ nos sistemas de produção com a adubação orgânica. Nas camadas de 0-10 e 10-20 cm, os maiores estoques foram observados nos sistemas $\mathrm{AO}$ $\left(1,78 \mathrm{Mg} \mathrm{ha}^{-1}\right)$ e AM2 +AO (1,56 $\left.\mathrm{Mg} \mathrm{ha}^{-1}\right)$, respectivamente (Quadro 6). A manutenção dos estoques de C do solo, especial mente as frações lábeis, é essencial à mel horia da qualidade do solo e sustentabilidade destes sistemas de produção (Blair, 2000).

Os estoques de $C_{L}$ na $F A$ foram maiores do que nos sistemas agrícolas na camada de $0-10 \mathrm{~cm}$, similarmente ao constatado por Hu et al. (1997). A redução nos estoques de $C_{L}$ em relação à $F A$ foi de 49 e 19 \% nos sistemas sem adubação orgânica (0, AM1 e AM2) e com adubação orgânica (AO, $\mathrm{AM} 1$ + AO eAM 2 +AO), respectivamente. Por outro lado, na camada de $10-20 \mathrm{~cm}$, apenas os sistemas quenãor receberama adubação orgânica apresentaram redução nos estoques de $C_{L}$ (17\%), (Quadro 6). Nos sistemas com adubação orgânica, observou-se aumento médio dos estoques de $C_{L}$ em relaçãoà $F A$, de $29 \%$, o que diferenciou este compartimento do $\mathrm{C}_{\text {MIC }}$ e do $\mathrm{C}_{\mathrm{FL}}$, que apresentaram redução nos estoques em todas camadas e sistemas estudados. Os estoques de $C_{L}$ declinaram rapidamente, porém a recuperação destes estoques foi similarmente

Quadro 6. Estoques de carbono lábil $\left(C_{\mathrm{L}}\right)$ e de carbono não-lábil $\left(\mathrm{C}_{\mathrm{NL}}\right)$ nas profundidades de 0-10, 10-20 e 0-20 cm, de um Argissolo Vermelho-Amarelo, de acordo com as adubações, mineral e orgânica, e em Floresta Atlântica

\begin{tabular}{|c|c|c|c|c|c|c|}
\hline \multirow{2}{*}{ Adubo mineral } & \multicolumn{2}{|c|}{ Adubo orgânico } & \multirow{2}{*}{ Média } & \multicolumn{2}{|c|}{ Adubo orgânico } & \multirow{2}{*}{ Média } \\
\hline & 0 & AO & & $\mathbf{0}$ & AO & \\
\hline & \multicolumn{2}{|c|}{$-C_{L}, M g ~ h a-1$} & & \multicolumn{2}{|c|}{$-\mathrm{C}_{\mathrm{NL}}, \mathrm{Mg} \mathrm{ha}^{-1}$} & \\
\hline & & & $0 \mathrm{~cm}$ & & & \\
\hline FA & \multicolumn{2}{|c|}{$2,10 \pm 0,02$} & & \multicolumn{2}{|c|}{$32,90 \pm 2,42$} & \\
\hline 0 & $1,06 \mathrm{~b}$ & 1,78 a & 1,42 & $17,81 \mathrm{~b}$ & 23,56 a & 20,68 \\
\hline AM 1 & $1,02 \mathrm{~b}$ & 1,62 a & 1,32 & $20,63 \mathrm{~b}$ & 24,89 a & 22,76 \\
\hline AM 2 & $1,12 b$ & 1,69 a & 1,40 & $19,49 \mathrm{~b}$ & $24,23 a$ & 21,86 \\
\hline \multicolumn{7}{|c|}{$10-20 \mathrm{~cm}$} \\
\hline FA & \multicolumn{2}{|c|}{$1,18 \pm 0,04$} & & \multicolumn{2}{|c|}{$26,08 \pm 1,39$} & \\
\hline 0 & $1,10 \mathrm{~b}$ & 1,47 a & 1,28 & $16,94 \mathrm{~b}$ & $21,25 \mathrm{a}$ & 19,09 \\
\hline AM 1 & $0,95 \mathrm{~b}$ & 1,55 a & 1,25 & $17,17 b$ & $23,26 a$ & 21,21 \\
\hline AM 2 & $0,88 \mathrm{~b}$ & $1,56 \mathrm{a}$ & 1,22 & $18,50 \mathrm{~b}$ & $22,58 \mathrm{a}$ & 20,54 \\
\hline \multicolumn{7}{|c|}{$0-20 \mathrm{~cm}$} \\
\hline FA & \multicolumn{2}{|c|}{$3,43 \pm 0,06$} & \multicolumn{4}{|c|}{$60,17 \pm 4,32$} \\
\hline 0 & $2,16 b$ & $3,25 \mathrm{a}$ & 2,70 & $34,76 \mathrm{a}$ & $44,81 \mathrm{a}$ & 39,78 \\
\hline AM 1 & $1,97 \mathrm{~b}$ & $3,17 \mathrm{a}$ & 2,57 & $37,74 \mathrm{a}$ & 48,13 a & 42,93 \\
\hline AM 2 & $2,00 \mathrm{~b}$ & 3,25 a & 2,62 & 37,99 a & 46,80 a & 42,39 \\
\hline
\end{tabular}

Médias seguidas de mesma letra minúscula, na linha, não diferem entre si a 5 \% (teste F). FA: Floresta Atlântica; 0: testemunha; AM 1: $250 \mathrm{~kg} \mathrm{ha}^{-1}$ de 4-14-8 + $20 \mathrm{~kg} \mathrm{ha}^{-1}$ de $\mathrm{N}$ em cobertura; AM2: $500 \mathrm{~kg} \mathrm{ha}^{-1}$ de 4-14-8 + $40 \mathrm{~kg} \mathrm{ha}^{-1}$ de $\mathrm{N}$ em cobertura; $\mathrm{AO}$ $40 \mathrm{~m}^{3} \mathrm{ha}^{-1}$. 
Quadro 7. Proporção de car bono lábil no carbono orgânico total $\left(C_{L} / C O T\right)$ e Índice de Manejo de Carbono (IMC) nas profundidades de 0-10, 10-20 e 0-20 cm, de um Argissolo Vermel ho-Amarelo, de acordo com as adubações, mineral e orgânica, e em Floresta Atlântica

\begin{tabular}{|c|c|c|c|c|c|}
\hline \multirow{2}{*}{ Sistema } & \multirow{2}{*}{$\mathrm{C}_{\mathrm{L}} / \mathrm{COT}$} & \multicolumn{4}{|c|}{ Índice(1) } \\
\hline & & ICC & $\mathbf{L}$ & IL & IMC \\
\hline \multicolumn{6}{|c|}{$0-10 \mathrm{~cm}$} \\
\hline $\begin{array}{l}F A \\
0 \\
A M 1 \\
A M 2 \\
A O \\
A M 1+A O \\
A M 2+A O\end{array}$ & $\begin{array}{l}6,00 \\
5,62 \\
4,73 \\
5,42 \\
7,04 \\
6,10 \\
6,50\end{array}$ & $\begin{array}{l}0,52 \\
0,62 \\
0,57 \\
0,69 \\
0,71 \\
0,70\end{array}$ & $\begin{array}{l}0,06 \\
0,06 \\
0,05 \\
0,06 \\
0,08 \\
0,06 \\
0,07\end{array}$ & $\begin{array}{l}0,93 \\
0,78 \\
0,90 \\
1,18 \\
1,01 \\
1,09\end{array}$ & $\begin{array}{l}49 \\
48 \\
51 \\
81 \\
72 \\
76\end{array}$ \\
\hline $\begin{array}{l}F A \\
0 \\
A M 1 \\
A M 2 \\
A O \\
A M 1+A O \\
A M 2+A O\end{array}$ & $\begin{array}{l}4,34 \\
6,09 \\
5,24 \\
4,55 \\
6,47 \\
6,26 \\
6,47\end{array}$ & $\begin{array}{l}0,80 \\
0,87 \\
0,89 \\
1,01 \\
1,09 \\
1,06\end{array}$ & $\begin{array}{l}0,04 \\
0,06 \\
0,05 \\
0,05 \\
0,07 \\
0,07 \\
0,07\end{array}$ & $\begin{array}{l}1,44 \\
1,23 \\
1,06 \\
1,54 \\
1,48 \\
1,54\end{array}$ & $\begin{array}{r}116 \\
107 \\
94 \\
155 \\
162 \\
163\end{array}$ \\
\hline $\begin{array}{l}F A \\
0 \\
A M 1 \\
A M 2 \\
A O \\
A M 1+A O \\
A M 2+A O\end{array}$ & $\begin{array}{l}5,36 \\
5,85 \\
4,97 \\
5,00 \\
6,77 \\
6,18 \\
6,49\end{array}$ & $\begin{array}{l}0,63 \\
0,72 \\
0,69 \\
0,81 \\
0,86 \\
0,83\end{array}$ & $\begin{array}{l}0,06 \\
0,06 \\
0,05 \\
0,05 \\
0,07 \\
0,07 \\
0,07\end{array}$ & $\begin{array}{l}1,09 \\
0,92 \\
0,92 \\
1,27 \\
1,16 \\
1,22\end{array}$ & $\begin{array}{r}69 \\
66 \\
64 \\
103 \\
99 \\
102\end{array}$ \\
\hline
\end{tabular}

0: testemunha; AM 1: $250 \mathrm{~kg} \mathrm{ha}^{-1}$ de 4-14-8 + $20 \mathrm{~kg} \mathrm{ha}^{-1}$ de $\mathrm{N}$ em cobertura; $\mathrm{AM} 2: 500 \mathrm{~kg} \mathrm{ha}^{-1}$ de 4-14-8 + 40 kg ha-1 de $\mathrm{N}$ em cobertura; AO: adubo orgânico, $40 \mathrm{~m}^{3}$ ha-1; FA: Floresta Atlântica.

(1) ICC- Índice de compartimento de carbono; L- Labilidade; IL- Í ndice de Labilidade.

acelerada, o que o torna indicador sensível da dinâmica do C no sistema (Whitbread et al., 2000).

O índice de manejo de carbono (I MC) aumentou com a incorporação do composto orgânico (Quadro 7). $\mathrm{Na}$ camada de 0-20 cm, ol MC variou de 64 (AM 1) a 103 (AO). Valores de IMC inferiores a 100 são indicativos de impacto negativo das práticas de manejo sobre os teores da matéria orgânica e qualidade do solo (Blair et al., 1995). Assim, todos os sistemas contribuíram para redução dos estoques de COT na camada de 0-10 cm eaumento na camada de $10-20 \mathrm{~cm}$. Noentanto, os sistemas com a presença de adubação orgânica apresentaram maior preservação dos estoques de COT.

\section{CONCLUSÕES}

1. Os estoques de carbono orgânico e nitrogênio total diminuíram após a mudança de Floresta Atlântica para agricultura.

2. A presença de adubação orgânica aumentou os estoques de carbono orgấnico e nitrogênio total, em relação aos sistemas de produção com adubação mineral ou mesmo sem adubação, o que a posiciona como uma estratégia de manejo importante à conservação da qualidade do solo.

3. Os estoques do carbono da fração leve e do carbono lábil foram reduzidos mais intensamente do que o carbono orgânico total, especialmente nos sistemas de produção sem adubação orgânica, razão por que podem ser considerados indicadores mais sensíveis das mudanças no estado da matéria orgânica do solo.

4. Os sistemas de produção com a presença da adubação orgânica não apresentaram potencial para seqüestro e emitiram as maiores quantidades de C$\mathrm{CO}_{2}$ para atmosfera. No entanto, a aplicação do composto orgânico constituiu uma efetiva forma de reciclagem de nutrientes e retorno de $\mathrm{C}$ ao solo.

\section{LITE RATURA CITADA}

ANGERS, D.A.; BOLINDER, M.A.; CARTER, M.R.; GREGORICH, E.G.; DRURY, C.F.; LIANG, B.C.; VORONEY, R.P.; SIMARD, R.R.; DONALD, R.G.; BEYAERT, R.P. \& MARTEL, J . I mpact of tillage practices on organic carbon and nitrogen storage in cool, humid soils of eastern Canada. Soil Till. Res., 41:191-201, 1997. 
BLAIR N. Impact of cultivation and sugar-cane green trash management on carbon fractions and aggregate stability for a Chromic Luvisol in Queensland, Australia. Soil Till. Res., 55:183-191, 2000.

BLAIR. G.J .; LEFROY, R.D.B. \& LISLE, L. Soil carbon fractions based on their degree of oxidation, and development of a carbon management index for agricultural systems. Aust. J . Agric. Res., 46:1459-1466, 1995.

BREMNER, J .M. Nitrogen total. In: SPARKS, D.L., ed. Methods of soil analysis. Part 3. Madison, America Society of Agronomy, 1996. p.1085-1121 (SSSA Book Series, 5)

CARTER, M.R.; GREGORICH, E.G.; ANGERS, D.A.; DONALD, R.G. \& BOLINDER, M.A. Organic $C$ and $N$ storage and organic fractions, in adjacent cultivated and forested soils of eastern Canada. Soil Till. Res., 47:253-261, 1998.

FERNANDES, E.C.; MOTAVALLI, P.P.; CASTILLA, C. \& MUKURUMBIRA, L. Management control of soil organic matter dynamics in tropical land-use systems. Geoderma, 79:49-67, 1997.

FERREIRA, L.M. As interações entrea fração mineral ea fração orgânica em solos da região de Bauru-SP. São Paulo, Universidade deSão Paulo, 1997. 217p. (TesedeDoutorado)

GALVÃO, J.C.C. Características físicas e químicas do solo e produção de milho exclusivo e consorciado com feijão em função de adubações orgânica e mineral contínuas. Viçosa, Universidade Federal de Viçosa, 1995. 194p. (Tese de Doutorado)

GALVÃO, J .C.C. E feito das adubações orgânica e mineral sobre o consórcio milho-feijão. Viçosa, Universidade Federal de Viçosa, 1988. 112p. (Tese de Mestrado)

GREGORICH, E.G. \& J ANZEN, M.H. Storage of soil carbon in the light fraction and macro organic matter. In: CARTER, M.R. \& STEWART, B.A., eds. Advances in soil science. Structure and organic matter storage in agricultural soils. Boca Raton, CRC Lewis, 1996. p.167-385.

HOUGHTON, R.A.; SKOLE. D.L. \& LEFKOWITZ, D.S. Changes in the landscape of Latin America between 1850 and 1985. II Net release of $\mathrm{CO}_{2}$ to the atmosphere. For. Ecol. Manag., 38:173-199,1991.

HU, S.; COLEMAN, D.C.; CARROL, C.R.; HENDRIX, P.F. \& BEARE, M.H. Labile soil carbon pools in subtropical forest and agricultural ecosystems as influenced by management practices and vegetation types. Agric. Ecosys. Environ., 65:69-78, 1997

ISLAM, K.R. \& WEIL, R.R. Microwave irradiation of soil for routine measurement of microbial biomass carbon. Biol Fert. Soils.,27:408-416, 1998.

J ENKINSON, D.S. The Rothamsted long-term experiments: are they still of use? Agron. J ., 83:2-10, 1991.

KANCHIKERIMATH, M. \& SINGH, D. Soil organic matter and biological properties after 26 years of maize-wheat-cowpea cropping as affected by manure and fertilization in a Cambissol in semiarid region of India. Agric. Ecosys. Environ., 86:155-162, 2001.

KAPKIYAI, J .J .; KARANJ A, N.K.; QURESHI, J .N.; SMITHSON, P.C. \& WOOMER, P.L. Soil organic matter and nutrient dynamics in a Kenyan nitisol under long-term fertilizer and organic input management. Soil Biol. Biochem., 31:1773-1782, 1999.
LEITE, L.F.C. Compartimentos e dinâmica da matéria orgânica do sol o sob diferentes manejos e sua simulação pel o modelo Century. Viçosa Universidade Federal de Viçosa, 2002. 146p. (Tese de Doutorado)

LONGO, R.M. \& ESPÍNDOLA, C.R. C-orgânico, N-total e substâncias húmicas sob influência da introdução de pastagens (Brachiaria sp.) em áreas de Cerrado e F loresta Amazônica. R. Bras. Ci. Solo, 24:723-729, 2000.

LUTZOW, M. von; LEIFELD, J .; KAINZ, M.; KNABNERKOGEL, I. \& MUNCH, J.C. Indications for soil organic matter quality in soils under different management. Geoderma, 105:243-258, 2002.

MANFOGOYA, P.L.; DZOWELA, B.H. \& NAIR, P.K. Effect of multipurpose trees, age of cutting and drying method on pruning quality. In: CADISCH, G. \& GILLER, K.E., eds. Driven by nature: plant litter quality and decomposition. Wallingford CAB, International, 1997. p.167-174.

OMAY, A.B.; RICE, C.W.; MADDUX, L.D. \& GORDON, W.B. Changes in soil microbial and chemical properties under long-term crop rotation and fertilization. Soil Sci. Soc. Am. J ., 61:1672-1678, 1997.

PALM, C.A.; GACHENKO, C.N.; DELVE, R.J .; CADISCH, G. \& GILLER, K.E. Organic inputs for soil fertility management in tropical agroecosystems: application of organic resource database. Agri. Ecosys. Environ., 83:27-42, 2001.

PARTON, W.J .; SCHIMEL, D.S.; COLE, C.V. \& OJIMA, D.S. Analysis of factors controlling soil organic matter levels in great plains grasslands. Soil Sci. Soc. Am. J ., 51:1173-1179, 1987.

PAUSTIAN, K.; ANDRÉN, O.; J ANZEN, H.H.; LAL, R.; SMITH, P.; TIAN, G.; TIESSEN, H.; Van NOORDWIJ K, M. \& WOOMER, P. Agricultural soils as a sink to mitigate $\mathrm{CO}_{2}$ emissions. Soil Use Manag., 13:230-244, 1997.

ROSALES, M.A.; OLIVEIRA, O.S.; MOURA, M.A. \& LOURES, E.G. I nfluência das adubações orgânica e mineral contínuas sobre as características das frações das substâncias húmicas do solo. R. Ceres, 46:67-81, 1999.

SCHOLES, M.C.; POWLSON, D. \& TIAN, G. Input control of organic matter dynamics. Geoderma, 79:25-47, 1997.

SHANG, C. \& TIESSEN, H. Organic matter lability in a tropical oxisol: evidence from shifting cultivation, chemical oxidation, particle size, density, and magnetic fractionations. Soil Sci., 162:795-807, 1997.

SIX, J .; ELLIOT E.T.; PAUSTIAN, K. \& DORAN, J.W. Aggregation and soil organic matter accumulation in cultivated and native grassland soils. Soil Sci Soc. Am. J ., 62:1153-1161, 1998

SIX, J .; MERCKX, R.; KIMPE, K.; PAUSTIAN, K. \& ELLIOT, E.T. A re-evaluation of the enriched labile soil organic matter fraction. Eur. J . Soil Sci., 51:283-293, 2000.

SOHI, S.; MAHIEU, N.; ARAH, J .R.M.; POLWSON, D.S.P.; MADARI, B. \& GAUNT, J .L. A procedure for isolating soil organic matter fractions suitable for modeling. Soil Sci. Soc. Am. J ., 65:1121-1128, 2001.

SPARLING, G.P. Ratio of microbial biomass carbon to soil organic carbon as a sensitive indicator of changes in soil organic matter. Aust. J . Soil Res., 30:195-207, 1992. 
SPARLING, G.P. \& WEST, A.W. A direct extraction method to estimate soil microbial C:Calibration in situ using microbial respiration and ${ }^{14} \mathrm{C}$ labelled cells. Soil Biol. Biochem., 20:337-343, 1988.

STEVENSON, F.J . Humus chemistry. New York, J ohn Wiley \& Sons, 1994. 496p.

SWIFT, R.S. Organic matter characterization. In: SPARKS, D.L., ed. Methods of soil analysis. Part 3. Madison, America Society of Agronomy, 1996. p.1011-1020 (SSSA Book Series, 5)

SWIFT, R.S. Sequestration of carbon by soil. Soil Sci., 166:858871, 2001.

UNIVERSIDADE FEDERAL DE VIÇOSA - UFV. SAEGSistema para análise estatística v.5.0. Viçosa, 1993.
WHITBREAD, A.M.; BLAIR, G.J . \& LEFROY, R.D.B. Managing legume leys, residues and fertilizers to enhance the sustainability of wheat cropping systems in Australia 2. Soil physical fertility and carbon. Soil Till. Res., 54:77-89, 2000.

WITTER, E. \& KANAL, A. Characteristics of the soil microbial biomass in soils from a long-term field experiment with different levels of C input. Appl. Soil Ecol., 10:37-49, 1998.

YEOMANS, J.C. \& BREMNER, J .M. A rapid and precisemethod for routine determination of organic carbon in soil. Comm. Soil Sci. Plant Anal., 19:1467-1476, 1988.

ZECH, W.; SENESI, N.; GUGGENBERGER, G.; KAISER, K.; LEHMANN, J .; MIANO, T.M.; MILTNER, A. \& SCHROTH, G. Factor controlling humification and mineralization of soil organic matter in the tropics. Geoderma, 79:117-161, 1997. 